\title{
The voter's guide to the galaxy — a multiple-criteria fuzzy decision- support tool for voters and a fresh take on election survey methods
}

\author{
Sofia Maria Panzeri \\ Universitá degli Studi di Milano, \\ Via Festa del Perdono 7, 20122 \\ Milano, Italy, and \\ Palacky University Olomouc, Faculty \\ of Arts, Department of Economic and \\ Managerial studies, Email: \\ sofiamaria.panzeri@studenti.unimi.it
}

\author{
Jana Stoklasová \\ School of Business and \\ Management, LUT University, \\ Yliopistonkatu 34, \\ 53851 Lappeenranta, Finland \\ Email: jana.stoklasova@lut.fi
}

\author{
Jan Stoklasa \\ School of Business and \\ Management, LUT University, \\ Yliopistonkatu 34, \\ 53851 Lappeenranta, Finland, and \\ Palacky University Olomouc, \\ Faculty of Arts, Department of \\ Economic and Managerial Studies \\ Email: jan.stoklasa@lut.fi \\ Email: jan.stoklasa@upol.cz
}

\begin{abstract}
This paper suggests a multiple-criteria decisionsupport tool for voters, that compares the attitudes of the voters with the declared attitudes of the political parties in several sets of relevant issues. The model intends to identify parties that seem to provide the best fit with the voter attitude-wise. The data input methodology uses discrete 5-point Likert-type scales. We investigate the effect of the inclusion of weights of different sets of issues, of the numerical anchors of the values of the Likert-type scales and also of the potential presence of extremity/leniency effect on the suggestion of the "most compatible" political party suggestion. We also propose a simple fuzzyrule based evaluation tool to identify serious incompatibilities or desired compatibilities in the attitudes of the voter and the party to the relevant issues. This tool introduces (un)acceptability thresholds for the differences in attitudes between the parties and the respondents and provides lists of parties to vote for or to avoid voting for accompanied by the strengths of these suggestions. The tool is shown to have several desirable features including lower sensitivity to small differences in the attitudes, respondents' ability to express their preferences and also preventing the compensation of unacceptable differences in some categories of important issues by high compatibility in the other categories.
\end{abstract}

\section{INTRODUCTION}

$\mathrm{T}$ HE issue of elections is a topical one since the very beginning of democracy. It is becoming even more complex nowadays with increased (cyber) security concerns [1]. Choosing one's representatives or at least the political party that reflects one's values well enough is, however, a difficult problem to face. The choice of the most appropriate representing party would be a difficult one even if the voters had full information concerning the program, values, intentions, and goals of the parties/individuals to be chosen. In many cases, however, the assumption of full information is unachievable. In these cases, one might decide based on a sample of key issues and the similarity/difference of his/her attitudes towards these and the attitudes of the political parties. Ballot and voter decision-support systems are being dis- cussed and proposed to help voters get oriented in the vast amount of information available and to facilitate information management [2]. However, these are very information extensive and require a sufficient knowledge of their users and advanced knowledge of human-computer interaction. As such their introduction in practice might be difficult. Still a simpler and less advanced voter decision-support can be beneficial and constitute a step towards the desired integrated voter-support information systems.

This is exactly the point of departure of this paper. We assume that a voter intends to choose as rationally as possible. Rational choice in this case is operationalized as the act of choosing the party that expresses its opinions or attitudes to the key issues (or sets/groupings thereof) in the way most similar to one's own attitudes. Our question is how to decide what is "most similar" in this context - particularly in such a way that would be applicable for political and social sciences research and also for election surveys as well as for actual voter decision support. This means that we will be relying on the information available in the program statements of the parties and on expert assessment thereof, when needed. We will also be using simple tools for data input, namely Liker (type) scales [3].

In line with the finding of Rogowski [4] we assume that voters tend to vote for those parties that have similar (general) ideological orientations. This means that we can afford to focus on several key issues that overall capture the attitude (or ideology) of the party and of the voter to be supported by the proposed system. Several tools for the assessment of agreement of one's attitudes with those of others that deal with the closeness of the attitudes (including their uncertainty) in the semantic space are already available $[5,6]$. Even though various types of consensus have recently been proposed for these methods [7] and the attitudes can be represented with the corresponding uncertainty stemming from the data input method as well as from the nature of the decision-makers and the concepts being assessed, these 
methods require a more complex data input method than would be desirable in the context of voter decision-support with multiple key areas being considered.

In this paper we therefore suggest a method that is simpler in terms of data input, but still allows for the assessment of compatibility of one's own values with those declared by the parties in line with [4] and provides valid decision-support. Obviously, when the goal of simplicity of obtaining input data is set, there are drawbacks to be expected in the process of the analysis of the data. In this case we will discuss the effect of the calibration of the numerical values of discrete Likert scales with linguistic labels [8,9] and also the possibility of getting more insights or more real-life representation of the preferences, attitudes or values using the tools of fuzzy set theory [10]. We are well aware that some election surveys and popular voter "calculators" providing fast and popular "compatibility" suggestions to voters use a similar approach, but these are frequently using just a binary scale and do not offer any customizability. Our approach strives to allow for the reflection of different strengths of support/opposition concerning a specific issue and thus on different magnitudes of differences in the attitudes to the selected crucial issues.

\section{PROBLEM DEFINITION AND CONTEXT}

In this paper we assume the perspective of 5 young potential Italian voters represented by actual respondents, and we set the goal of identifying the most "fitting" party to vote for based on the compatibility (difference) of the attitudes of the respondents and declared attitudes of the parties.

The set of parties consists of eight Italian political parties:

- Movimento 5 Stelle (“anti-establishment”)

- Lega (right wing)

- Partito Democratico (centre-left)

- Forza Italia (centre-right)

- Fratelli d'Italia (far right wing)

- Italia Viva (centre-left)

- Liberi e Uguali (centre-left)

- $\quad$ Europa e Azione (centre-left)

The above listed parties serve as real-life examples of parties, are selected so that they represent different declared attitudes to the selected crucial issues and at the same time allow the assessment of the reasonability of the provided voter decision-support and its sensitivity to the calibration of the used scales. We are not assuming a position of support/opposition with respect to any of these parties. The summary labels of the parties provided in the brackets are intended as "guides to the understanding of the overall philosophy/ideology of the party", they have been assigned by the authors of the paper and might constitute a large simplification of the actual goals and attitudes of the party. Nevertheless, we think that since this represents an example setting for the proposed method, the labels can provide the reader a better ability to assess the results of the decision support suggested in this paper.

\section{A. The important issues used to assess the compatibility between the respondents' attitudes and the attitudes of the political parties}

The crucial issues to be considered were compiled by the authors and in partial cooperation with the respondents with the aim to cover the most important areas as considered by the respondents. This is well in line with the idea of the use of the proposed framework as a voter decision-support tool. On the other hand, if an overall "attitude compatibility study" were to be conducted, then the list of the important issues can be compiled by the researcher in accordance with the needs and goals of the study. The crucial issues are grouped into 6 main categories. This allows for a detailed issue-by-issue attitudecompatibility analysis but also for a more complex (potentially repeated) assessment of the attitudes towards the overall issue categories. The considered issues and their categories are the following:

\section{C1. SOCIAL ISSUES}

C1,1 Are you for or against ABORTION?

$\mathrm{C} 1,2$ Are you for or against EUTHANASIA?

$\mathrm{C} 1,3$ Are you for or against the DEATH PENALTY?

C1,4 Are you for or against LGBTQIA+ ADOPTION RIGHTS?

C1,5 Are you for or against SAME SEX MARRIAGE?

\section{C2. FOREIGN POLICY ISSUES}

C2,1 Are you for or against ITALY'S WITHDRAW FROM THE EU?

C2,2 Are you for or against the GONVERNMENT INFLUENCING FOREIGN ELECTIONS?

C2,3 Are you for or against the UNITED STATES of EUROPE?

C2,4 Are you for or against an INCREASE in MANDATORY MILITARY SPENDING?

$\mathrm{C} 2,5$ Are you for or against the creation of an EU ARMY?

\section{C3. IMMIGRATION ISSUES}

C3,1 Are you for or against a TEMPORARY IMMIGRATION BAN?

C3,2 Are you for or against DEPORTING CRIMINAL IMMIGRANTS? (violent crimes)

C3,3 Are you for or against BANNING MUSLIMS IMMIGRANTS FROM ENTERING THE COUNTRY?

C3,4 Are you for or against an EU IMPOSED QUOTA OF MIGRANTS PER COUNTRY?

C3,5 Are you for or against IMMIGRANTS taking a CITIZENSHIP TEST?

\section{C4. HEALTHCARE ISSUES}

C4,1 Are you for or against the ISSUE of VACCINE PASSPORTS?

C4,2 Are you for or against an INCREASE in FUNDING for MENTAL HEALTH?

C4,3 Are you for or against the PRIVATIZATION of HOSPITALS? 
TABLE I.

ASSESSMENT OF THE ATTITUDES OF THE PARTIES TO THE J-TH ISSUE IN THE I-TH CATEGORY (REPRESENTED BY THE VALUE IN THE I-TH ROW AND J-TH COLUMN IN EACH RESPECTIVE MATRIX) UNDER DIFFERENT SETUPS OF THE LIKERT SCALE. SETUPS I AND II ARE STANDARD 5-POINT EQUIDISTANT LIKERT SCALE CODINGS, SETUP III IS 5-POINT NON-EQUIDISTANT BUT SYMMETRICAL LIKERT SCALE CODING AND SETUP IV IS A 3-POINT SCALE ANALOGY TO STOKLASA ET. AL. [9]. EXAMPLE OF A RESULT OF EXPERT ASSESSMENT.

SETUP I and II

MOVIMENTO 5 STELLE

\begin{tabular}{|c|c|c|c|c|}
\hline 2 & 1 & 5 & 2 & 2 \\
\hline 4 & 5 & 5 & 4 & 4 \\
\hline 4 & 1 & 5 & 1 & 1 \\
\hline 2 & 2 & 5 & 2 & 1 \\
\hline 1 & 4 & 3 & 5 & 1 \\
\hline 5 & 5 & 2 & 3 & 1 \\
\hline
\end{tabular}

LEGA
\begin{tabular}{|c|c|c|c|c|}
\hline 2 & 3 & 1 & 5 & 4 \\
\hline 4 & 5 & 5 & 1 & 4 \\
\hline 1 & 1 & 1 & 1 & 1 \\
\hline 2 & 2 & 1 & 5 & 5 \\
\hline 1 & 4 & 1 & 1 & 4 \\
\hline 2 & 5 & 5 & 4 & 1 \\
\hline
\end{tabular}
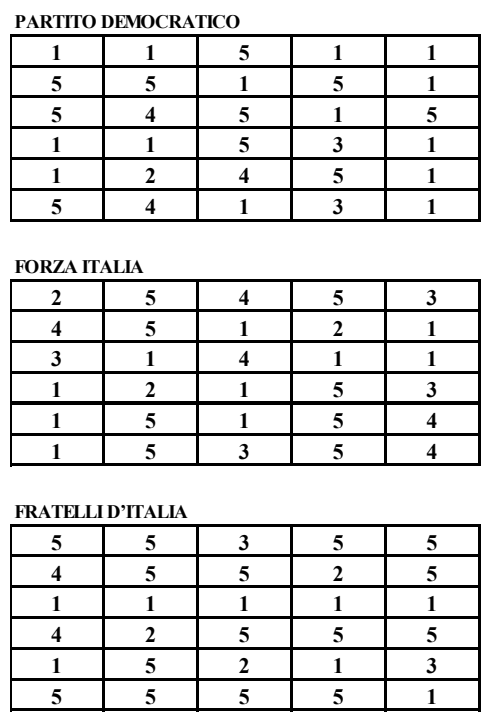

ITALIA VIVA

\begin{tabular}{|l|l|l|l|l|}
\hline 1 & 2 & 5 & 1 & 2 \\
\hline 5 & 5 & 1 & 3 & 2 \\
\hline 3 & 3 & 4 & 4 & 2 \\
\hline 2 & 1 & 4 & 3 & 2 \\
\hline 1 & 2 & 2 & 4 & 4 \\
\hline 4 & 2 & 3 & 4 & 4 \\
\hline
\end{tabular}

LIBERI E UGUALI
\begin{tabular}{|c|c|c|c|c|}
\hline 1 & 1 & 5 & 1 & 1 \\
\hline 5 & 5 & 1 & 4 & 1 \\
\hline 5 & 5 & 5 & 1 & 5 \\
\hline 1 & 1 & 5 & 1 & 1 \\
\hline 1 & 1 & 5 & 5 & 1 \\
\hline 5 & 5 & 1 & 2 & 1 \\
\hline
\end{tabular}

+EUROPA and AZIONE
\begin{tabular}{|c|c|c|c|c|}
\hline 1 & 1 & 5 & 1 & 1 \\
\hline 5 & 5 & 1 & 5 & 1 \\
\hline 5 & 5 & 5 & 1 & 5 \\
\hline 1 & 1 & 5 & 1 & 1 \\
\hline 1 & 3 & 4 & 5 & 1 \\
\hline 5 & 4 & 1 & 2 & 1 \\
\hline
\end{tabular}

\section{SETUP III}

MOVIMENTO 5 STELLE
\begin{tabular}{|c|c|c|c|c|}
\hline 2.5 & 1 & 5 & 2.5 & 2.5 \\
\hline 3.5 & 5 & 5 & 3.5 & 3.5 \\
\hline 3.5 & 1 & 5 & 1 & 1 \\
\hline 2.5 & 2.5 & 5 & 2.5 & 1 \\
\hline 1 & 3.5 & 3 & 5 & 1 \\
\hline 5 & 5 & 2.5 & 3 & 1 \\
\hline
\end{tabular}

LEGA
\begin{tabular}{|c|c|c|c|c|}
\hline 2.5 & 3 & 1 & 5 & 3.5 \\
\hline 3.5 & 5 & 5 & 1 & 3.5 \\
\hline 1 & 1 & 1 & 1 & 1 \\
\hline 2.5 & 2.5 & 1 & 5 & 5 \\
\hline 1 & 3.5 & 1 & 1 & 3.5 \\
\hline 2.5 & 5 & 5 & 3.5 & 1 \\
\hline
\end{tabular}

PARTITO DEMOCRATICO
\begin{tabular}{|c|c|c|c|c|}
\hline 1 & 1 & 5 & 1 & 1 \\
\hline 5 & 5 & 1 & 5 & 1 \\
\hline 5 & 3.5 & 5 & 1 & 5 \\
\hline 1 & 1 & 5 & 3 & 1 \\
\hline 1 & 2.5 & 3.5 & 5 & 1 \\
\hline 5 & 3.5 & 1 & 3 & 1 \\
\hline
\end{tabular}

FORZA ITALIA
\begin{tabular}{|c|c|c|c|c|}
\hline 2.5 & 5 & 3.5 & 5 & 3 \\
\hline 3.5 & 5 & 1 & 2.5 & 1 \\
\hline 3 & 1 & 3.5 & 1 & 1 \\
\hline 1 & 2.5 & 1 & 5 & 3 \\
\hline 1 & 5 & 1 & 5 & 3.5 \\
\hline 1 & 5 & 3 & 5 & 3.5 \\
\hline
\end{tabular}

FRATELLID'TAALIA
\begin{tabular}{|c|c|c|c|c|}
\hline 5 & 5 & 3 & 5 & 5 \\
\hline 3.5 & 5 & 5 & 2.5 & 5 \\
\hline 1 & 1 & 1 & 1 & 1 \\
\hline 3.5 & 2.5 & 5 & 5 & 5 \\
\hline 1 & 5 & 2.5 & 1 & 3 \\
\hline 5 & 5 & 5 & 5 & 1 \\
\hline
\end{tabular}

\section{ITALIA VIVA}

\begin{tabular}{|c|c|c|c|c|}
\hline 1 & 2.5 & 5 & 1 & 2.5 \\
\hline 5 & 5 & 1 & 3 & 2.5 \\
\hline 3 & 3 & 3.5 & 3.5 & 2.5 \\
\hline 2.5 & 1 & 3.5 & 3 & 2.5 \\
\hline 1 & 2.5 & 2.5 & 3.5 & 3.5 \\
\hline 3.5 & 2.5 & 3 & 3.5 & 3.5 \\
\hline
\end{tabular}

LIBERI E UGUALI
\begin{tabular}{|c|c|c|c|c|}
\hline 1 & 1 & 5 & 1 & 1 \\
\hline 5 & 5 & 1 & 3.5 & 1 \\
\hline 5 & 5 & 5 & 1 & 5 \\
\hline 1 & 1 & 5 & 1 & 1 \\
\hline 1 & 1 & 5 & 5 & 1 \\
\hline 5 & 5 & 1 & 2.5 & 1 \\
\hline
\end{tabular}

+EUROPA and AZIONE
\begin{tabular}{|c|c|c|c|c|}
\hline 1 & 1 & 5 & 1 & 1 \\
\hline 5 & 5 & 1 & 5 & 1 \\
\hline 5 & 5 & 5 & 1 & 5 \\
\hline 1 & 1 & 5 & 1 & 1 \\
\hline 1 & 3 & 3.5 & 5 & 1 \\
\hline 5 & 3.5 & 1 & 2.5 & 1 \\
\hline
\end{tabular}

SETUP IV

MOVIMENTO 5 STELLE
\begin{tabular}{|c|c|c|c|c|}
\hline 1 & 1 & 5 & 1 & 1 \\
\hline 5 & 5 & 5 & 5 & 5 \\
\hline 5 & 1 & 5 & 1 & 1 \\
\hline 1 & 1 & 5 & 1 & 1 \\
\hline 1 & 5 & 3 & 5 & 1 \\
\hline 5 & 5 & 1 & 3 & 1 \\
\hline
\end{tabular}

LEGA
\begin{tabular}{|c|c|c|c|c|}
\hline 1 & 3 & 1 & 5 & 5 \\
\hline 5 & 5 & 5 & 1 & 5 \\
\hline 1 & 1 & 1 & 1 & 1 \\
\hline 1 & 1 & 1 & 5 & 5 \\
\hline 1 & 5 & 1 & 1 & 5 \\
\hline 1 & 5 & 5 & 5 & 1 \\
\hline
\end{tabular}

PARTITO DEMOCRATICO
\begin{tabular}{|c|c|c|c|c|}
\hline 1 & 1 & 5 & 1 & 1 \\
\hline 5 & 5 & 1 & 5 & 1 \\
\hline 5 & 5 & 5 & 1 & 5 \\
\hline 1 & 1 & 5 & 3 & 1 \\
\hline 1 & 1 & 5 & 5 & 1 \\
\hline 5 & 5 & 1 & 3 & 1 \\
\hline
\end{tabular}

FORZA ITALIA
\begin{tabular}{|c|c|c|c|c|}
\hline 1 & 5 & 5 & 5 & 3 \\
\hline 5 & 5 & 1 & 1 & 1 \\
\hline 3 & 1 & 5 & 1 & 1 \\
\hline 1 & 1 & 1 & 5 & 3 \\
\hline 1 & 5 & 1 & 5 & 5 \\
\hline 1 & 5 & 3 & 5 & 5 \\
\hline
\end{tabular}

FRATELLD'TTALIA
\begin{tabular}{|c|c|c|c|c|}
\hline 5 & 5 & 3 & 5 & 5 \\
\hline 5 & 5 & 5 & 1 & 5 \\
\hline 1 & 1 & 1 & 1 & 1 \\
\hline 5 & 1 & 5 & 5 & 5 \\
\hline 1 & 5 & 1 & 1 & 3 \\
\hline 5 & 5 & 5 & 5 & 1 \\
\hline
\end{tabular}

ITALIA VIVA
\begin{tabular}{|c|c|c|c|c|}
\hline 1 & 1 & 5 & 1 & 1 \\
\hline 5 & 5 & 1 & 3 & 1 \\
\hline 3 & 3 & 5 & 5 & 1 \\
\hline 1 & 1 & 5 & 3 & 1 \\
\hline 1 & 1 & 1 & 5 & 5 \\
\hline 5 & 1 & 3 & 5 & 5 \\
\hline
\end{tabular}

LIBERI E UGUALI
\begin{tabular}{|c|c|c|c|c|}
\hline 1 & 1 & 5 & 1 & 1 \\
\hline 5 & 5 & 1 & 5 & 1 \\
\hline 5 & 5 & 5 & 1 & 5 \\
\hline 1 & 1 & 5 & 1 & 1 \\
\hline 1 & 1 & 5 & 5 & 1 \\
\hline 5 & 5 & 1 & 1 & 1 \\
\hline
\end{tabular}

+EUROPA and AZIONE
\begin{tabular}{|c|c|c|c|c|}
\hline 1 & 1 & 5 & 1 & 1 \\
\hline 5 & 5 & 1 & 5 & 1 \\
\hline 5 & 5 & 5 & 1 & 5 \\
\hline 1 & 1 & 5 & 1 & 1 \\
\hline 1 & 3 & 5 & 5 & 1 \\
\hline 5 & 5 & 1 & 1 & 1 \\
\hline
\end{tabular}


C4,4 Are you for or against the institution of SAFE HAVENS?

C4,5 Are you for or against the LEGALIZATION of MARIJUANA?

\section{C5. ECONOMIC ISSUES}

C5,1 Are you for or against the SAME SALARY for MEN and WOMEN for the SAME JOB?

C5,2 Are you for or against RAISING TAXES on the RICH?

C5,3 Are you for or against CUTS to PUBLIC SPENDING in order to REDUCE NATIONAL DEBT?

C5,4 Are you for or against an INCREASE on TARIFFS on PRODUCTS IMPORTED into the country?

C5,5 Are you for or against FEWER RESTRICTIONS on CURRENT WELFARE BENEFITS?

\section{C6. CRIMINAL ISSUES}

C6,1 Are you for or against the PRIVATIZATION of PRISONS?

C6,2 Are you for or against the RELEASE from JAIL of NON-VIOLENT PRISONERS? (to reduce overcrowding)

C6,3 Are you for or against CONVICTED CRIMINALS having the RIGHT TO VOTE?

C6,4 Are you for or against the DEFUNDING of the POLICE?

C6,5 Are you for or against passing laws which PROTECT WHISTLEBLOWERS?

In order to allow for some expression of the strength of support or opposition of a specific issue, Likert scales are used to obtain the assessment of the attitudes of the parties and also of the individual respondents (i.e. potential voters). For the purpose of this paper we first adopt a 5-point Likert scale with linguistic values "strongly for", "slightly for", "neutral", "slightly against" and "strongly against".

\section{B. Different configurations of the Likert scales used in the decision support tool}

To be able to perform calculations, we need to assign numerical values to the linguistic values of the scales. This step potentially introduces several methodological issues (see e.g. [9-13] for a more detailed discussion of some of them). In this research we are focusing on the reasonability of performing calculations with the numerical values of the scales [11] that is connected with the (non)equidistance of the used numerical meanings of the linguistic values of the scale [9] and the differences in the perception of the relative distances between the linguistic values as compared to the perceived distances of their numerical meanings. We also reflect the potential ambiguity of the linguistic terms and their different interpretation by different individuals that might result in a different numerical value being the appropriate meaning of the linguistic term for different individuals [10]. Last but not least we consider the effect of leniency/central tendency $[12,13]$ and apply an analogy to the 3-bin histogram based solution proposed in [9]. For this reason, we propose the use of the following configurations of the numerical meanings of the linguistic values of the Likert scales:

- Setups I and II assign integer values to the linguistic values. In other words, "strongly for", "slightly for", "neutral", "slightly against" and "strongly against" are represented by 1, 2, 3, 4 and 5 respectively. This setup uses the usual approach to Likert scales and considers the linguistic values equidistant meaning-wise. As the equidistance of the perceived meaning of the linguistic terms cannot be guaranteed, this assignment of numerical meanings to the linguistic values is questioned by some authors as the source of serious limitations for the subsequent reliable processing of these values. Nevertheless, this configuration is being used frequently in practice and as such it provides a good benchmark for the other proposed setups. Setups I and II use the same Likert scale configuration, Setup I considers all the categories of issues equally important, whereas Setup II assigns different weights to different categories.

- Setup III assumes a "calibration" of the numerical meanings of the linguistic terms of the Likers scale has been performed and as a result of it the values "slightly for" and "slightly against" are semantically closer to the "neutral" term than to their respective "strongly for" and "strongly against" counterparts. The numerical values used as meanings of the linguistic terms are 1, 2.5, 3, 3.5 and 5 for "strongly for", "slightly for", "neutral", "slightly against" and "strongly against" respectively. The use of this setup does not assume that the calibration of the values proposed here is valid universally. It is meant to show what could be the results of appropriate calibration of the meanings of the linguistic values (as stressed in $[14,15]$ for example) compared to the use of the standard use of Likert scales represented by Setup I. Note that the proposed calibration at least preserves the symmetry of the scale with respect to the mean value, which is required by Likert [3].

- Setup IV offers a possible solution to the presence of central tendency or leniency bias, that is to the tendency of some people to avoid extreme values of the scales or to prefer using these values respectively. In essence it can be argued that "strongly for" can be representing the same strength of support for one respondent as "slightly for" for another respondent. If this is the case, then assigning different numerical meanings (or treating these answers as different, even though they might represent an identical strength of support) can be incorrect. Stoklasa et al. suggest in [9] the use of aggregated $+/ 0 /$ - classes. This means that all positive answers are grouped in one class (denoted + ), all negative answers in another one (denoted -) and those that can be considered neutral in a third one (denoted 0 ). This can be achieved by representing "strongly for", "slightly for", "neutral", "slightly 
against" and "strongly against" by 1, 1, 3, 5 and 5 values respectively.

\section{Expert assessment of the political parties' attitudes towards the important issues}

Table I summarizes the assessment of the attitudes of the selected eight political parties to the chosen thirty important issues by an expert evaluator. The first column of matrices represents the numerical values corresponding with the standard configuration of the 5-point Likert scale (Setup I and II), the middle column of matrices represents the same linguistic assessments but transformed into numerical ones using a recalibrated scale (Setup III), the right column represents the same linguistic assessments as the previous two columns of matrices, just coded using the $+/ 0 /$ - configuration (Setup IV). In each matrix the element in the position $(i, j)$ represents the evaluation of the issue $C_{i, j}$, that is the evaluation of the $j$-th issue in the $i$-th category.

The presented values are an example of a possible assessment by an expert and do not need to fully reflect the actual attitudes declared by the parties. In a real-life setting the task of obtaining the assessments depicted in Table I could be performed by a group of domain experts. We will, however, consider them as representative of the parties' program declarations for the purpose of the calculations.

\section{Attitudes of the respondents}

The attitudes of the five respondents towards the thirty important issues were obtained using the 5-point Likert scales too and coded in accordance with the three above discussed Likert scale codings. Table II summarizes the results of this process. The respondents (we will call them Melania, Anna, Marco, Carlo and Sara) come from similar cultural and social backgrounds and for this reason the results of decision support for them can be expected to be similar to one another. Based on the expert assessment by the authors, we can conclude that most of the respondents are "close" to center-left parties and inclined to vote for parties closer to the left side of the spectrum.

TABLE II.

ASSESSMENT OF THE ATTITUDES OF THE RESPONDENTS TO THE J-TH ISSUE IN THE I-TH CATEGORY (REPRESENTED BY THE VALUE IN THE I-TH ROW AND J-TH COLUMN IN EACH RESPECTIVE MATRIX) UNDER DIFFERENT SETUPS OF THE LIKERT SCALE. SETUPS I AND II ARE STANDARD 5-POINT EQUIDISTANT LIKERT SCALE CODINGS, SETUP III IS 5-POINT NON-EQUIDISTANT BUT SYMMETRICAL LIKERT SCALE CODING AND SETUP IV IS A 3-POINT SCALE ANALOGY TO STOKLASA ET. AL. [9].

SETUP I and II

Melania
\begin{tabular}{|c|c|c|c|c|}
\hline 1 & 1 & 5 & 1 & 1 \\
\hline 5 & 5 & 2 & 3 & 2 \\
\hline 5 & 4 & 5 & 4 & 2 \\
\hline 1 & 1 & 5 & 1 & 1 \\
\hline 1 & 4 & 2 & 4 & 3 \\
\hline 5 & 4 & 1 & 4 & 1 \\
\hline
\end{tabular}

Anna
\begin{tabular}{|c|c|c|c|c|}
\hline 2 & 1 & 2 & 4 & 2 \\
\hline 4 & 4 & 2 & 4 & 4 \\
\hline 2 & 2 & 5 & 2 & 2 \\
\hline 2 & 2 & 4 & 2 & 1 \\
\hline 1 & 2 & 2 & 2 & 4 \\
\hline 5 & 2 & 2 & 4 & 2 \\
\hline
\end{tabular}

Marco
\begin{tabular}{|c|c|c|c|c|}
\hline 4 & 4 & 5 & 4 & 2 \\
\hline 5 & 5 & 1 & 3 & 1 \\
\hline 3 & 1 & 3 & 1 & 2 \\
\hline 2 & 2 & 4 & 4 & 1 \\
\hline 1 & 2 & 1 & 4 & 2 \\
\hline 5 & 4 & 1 & 4 & 4 \\
\hline
\end{tabular}

Carlo
\begin{tabular}{|c|c|c|c|c|}
\hline 2 & 1 & 5 & 2 & 1 \\
\hline 5 & 5 & 5 & 3 & 3 \\
\hline 5 & 5 & 5 & 3 & 2 \\
\hline 3 & 1 & 1 & 1 & 1 \\
\hline 1 & 3 & 2 & 3 & 1 \\
\hline 5 & 1 & 1 & 5 & 3 \\
\hline
\end{tabular}

Sara
\begin{tabular}{|c|c|c|c|c|}
\hline 1 & 1 & 4 & 1 & 1 \\
\hline 5 & 5 & 2 & 4 & 2 \\
\hline 5 & 1 & 5 & 1 & 1 \\
\hline 1 & 1 & 4 & 2 & 1 \\
\hline 1 & 1 & 3 & 4 & 3 \\
\hline 3 & 4 & 2 & 5 & 1 \\
\hline
\end{tabular}

SETUP III

Melania
\begin{tabular}{|c|c|c|c|c|}
\hline 1 & 1 & 5 & 1 & 1 \\
\hline 5 & 5 & 2.5 & 3 & 2.5 \\
\hline 5 & 3.5 & 5 & 3.5 & 2.5 \\
\hline 1 & 1 & 5 & 1 & 1 \\
\hline 1 & 3.5 & 2.5 & 3.5 & 3 \\
\hline 5 & 3.5 & 1 & 3.5 & 1 \\
\hline
\end{tabular}

Anna
\begin{tabular}{|c|c|c|c|c|}
\hline 2.5 & 1 & 2.5 & 3.5 & 2.5 \\
\hline 3.5 & 3.5 & 2.5 & 3.5 & 3.5 \\
\hline 2.5 & 2.5 & 5 & 2.5 & 2.5 \\
\hline 2.5 & 2.5 & 3.5 & 2.5 & 1 \\
\hline 1 & 2.5 & 2.5 & 2.5 & 3.5 \\
\hline 5 & 2.5 & 2.5 & 3.5 & 2.5 \\
\hline
\end{tabular}

Marco
\begin{tabular}{|c|c|c|c|c|}
\hline 3.5 & 3.5 & 5 & 3.5 & 2.5 \\
\hline 5 & 5 & 1 & 3 & 1 \\
\hline 3 & 1 & 3 & 1 & 2.5 \\
\hline 2.5 & 2.5 & 3.5 & 3.5 & 1 \\
\hline 1 & 2.5 & 1 & 3.5 & 2.5 \\
\hline 5 & 3.5 & 1 & 3.5 & 3.5 \\
\hline
\end{tabular}

Carlo
\begin{tabular}{|c|c|c|c|c|}
\hline 2.5 & 1 & 5 & 2.5 & 1 \\
\hline 5 & 5 & 5 & 3 & 3 \\
\hline 5 & 5 & 5 & 3 & 2.5 \\
\hline 3 & 1 & 1 & 1 & 1 \\
\hline 1 & 3 & 2.5 & 3 & 1 \\
\hline 5 & 1 & 1 & 5 & 3 \\
\hline
\end{tabular}

Sara
\begin{tabular}{|c|c|c|c|c|}
\hline 1 & 1 & 3.5 & 1 & 1 \\
\hline 5 & 5 & 2.5 & 3.5 & 2.5 \\
\hline 5 & 1 & 5 & 1 & 1 \\
\hline 1 & 1 & 3.5 & 2.5 & 1 \\
\hline 1 & 1 & 3 & 3.5 & 3 \\
\hline 3 & 3.5 & 2.5 & 5 & 1 \\
\hline
\end{tabular}

SETUP IV

Melania
\begin{tabular}{|c|c|c|c|c|}
\hline 1 & 1 & 5 & 1 & 1 \\
\hline 5 & 5 & 1 & 3 & 1 \\
\hline 5 & 5 & 5 & 5 & 1 \\
\hline 1 & 1 & 5 & 1 & 1 \\
\hline 1 & 5 & 1 & 5 & 3 \\
\hline 5 & 5 & 1 & 5 & 1 \\
\hline
\end{tabular}

Anna
\begin{tabular}{|c|c|c|c|c|}
\hline 1 & 1 & 1 & 5 & 1 \\
\hline 5 & 5 & 1 & 5 & 5 \\
\hline 1 & 1 & 5 & 1 & 1 \\
\hline 1 & 1 & 5 & 1 & 1 \\
\hline 1 & 1 & 1 & 1 & 5 \\
\hline 5 & 1 & 1 & 5 & 1 \\
\hline
\end{tabular}

Marco
\begin{tabular}{|c|c|c|c|c|}
\hline 5 & 5 & 5 & 5 & 1 \\
\hline 5 & 5 & 1 & 3 & 1 \\
\hline 3 & 1 & 3 & 1 & 1 \\
\hline 1 & 1 & 5 & 5 & 1 \\
\hline 1 & 1 & 1 & 5 & 1 \\
\hline 5 & 5 & 1 & 5 & 5 \\
\hline
\end{tabular}

Carlo
\begin{tabular}{|c|c|c|c|c|}
\hline 1 & 1 & 5 & 1 & 1 \\
\hline 5 & 5 & 5 & 3 & 3 \\
\hline 5 & 5 & 5 & 3 & 1 \\
\hline 3 & 1 & 1 & 1 & 1 \\
\hline 1 & 3 & 1 & 3 & 1 \\
\hline 5 & 1 & 1 & 5 & 3 \\
\hline
\end{tabular}

Sara
\begin{tabular}{|c|c|c|c|c|}
\hline 1 & 1 & 5 & 1 & 1 \\
\hline 5 & 5 & 1 & 5 & 1 \\
\hline 5 & 1 & 5 & 1 & 1 \\
\hline 1 & 1 & 5 & 1 & 1 \\
\hline 1 & 1 & 3 & 5 & 3 \\
\hline 3 & 5 & 1 & 5 & 1 \\
\hline
\end{tabular}




\section{DIFFERENT SETUPS OF THE DECISION SUPPORT TOOL AND THE SUGGESTIONS GENERATED BY THEM}

Before we introduce the different setups of the proposed decision support tool to be compared, we first need to introduce the in-model notation. Let us consider a set of eight political parties $P=\left\{P_{1}, \ldots, P_{8}\right\}$ and a set of five respondents $R=\left\{R_{1}, \ldots, R_{5}\right\}$. We also consider a set of six categories of important issues $C=\left\{C_{1}, \ldots, C_{6}\right\}$ each of which can be subdivided into five important issues relevant for the specific category, in other words $C_{i}=\left\{C_{i, 1}, \ldots, C_{i, 5}\right\}$ for all $i=1, \ldots, 6$. The assessment of the attitude of each party and respondent to each of the important issues is captured by the matrix $A^{r}=$ $\left\{a_{i, j}^{r}\right\}$, where $i=1, \ldots, 6, j=1, \ldots, 5$, and $r \in P$ for the parties or $r \in R$ for the respondents. The categories $C_{1}, \ldots, C_{6}$ can be assigned normalized weights $w_{1}, \ldots, w_{6}$ such that $w_{i} \geq 0$ for all $i=1, \ldots, 6$ and $\sum_{i=1}^{6} w_{i}=1$. It is even possible to assign respondent-specific weighting vectors. The vector of the possible numerical values representing the linguistic values of the 5-point Likert scale $L=$ ("strongly for", "slightly for", "neutral", "slightly against" and "strongly against") is denoted as $N=\left(n_{1}, \ldots, n_{5}\right)$. Therefore $a_{i, j}^{r} \in N$ for any $i, j$, and $r$.

\section{A. Setup I}

In this setup the standard coding of the Likert-scale linguistic values by subsequent integer (equidistant) values is used. This means that $N^{I}=(1,2,3,4,5)$. We also assume that all the categories $C_{1}, \ldots, C_{6}$ are considered equally important. We therefore do not need to define category weights for this purpose. As all the categories contain the same amount of important issues, we can define the difference between the attitudes expressed by a respondent $u \in R$ and attitudes of a party $v \in P$ simply as

$$
d^{I}\left(A^{u}, A^{v}\right)=\sum_{i=1}^{6} \sum_{j=1}^{5}\left|a_{i, j}^{u}-a_{i, j}^{v}\right|,
$$

where $a_{i, j}^{u}, a_{i, j}^{v} \in\{1,2,3,4,5\}$. This way the difference in attitudes is defined as the sum of differences in the numerical meanings of the linguistic terms of the Likert scales used to assess the thirty important issues. Given the fact that all the assessments use the same 5-point scale, the maximum possible difference can be defined as $d_{\max }^{I}=$ $30\left(\max \left(N^{I}\right)-\min \left(N^{I}\right)\right)=30(5-1)=120$. As such an absolute-type measure of compatibility of the attitudes of respondent $u \in R$ with the attitudes expressed by a party $v \in$ $P$ can be defined as

$$
c^{I}\left(A^{u}, A^{v}\right)=1-\frac{d^{I}\left(A^{u}, A^{v}\right)}{d_{\max }^{I}} .
$$

Clearly $c^{I}\left(A^{u}, A^{v}\right) \in[0,1]$ for any $u \in R$ and $v \in P$. $c^{I}\left(A^{u}, A^{v}\right)=1$ then means absolute $(100 \%)$ compatibility, or in other words, zero difference in attitudes expressed by the respondent and the party. The suggested party to vote for should then be such that maximizes the compatibility value for the given respondent. Under this approach we get the compatibilities summarized in Table III.
TABLE III.

SUMMARY OF THE COMPATIBILITY OF THE PREFERENCES EXPRESSED BY THE FIVE RESPONDENTS TO THE PREFERENCES EXPRESSED BY THE POLITICAL PARTIES UNDER SETUP I. FOR EACH RESPONDENT THE HIGHEST COMPATIBILITIES ARE DENOTED IN GREEN, THE LOWEST COMPATIBILITIES IN RED.

\begin{tabular}{lccccc} 
Setup I & Melania & Anna & Marco & Carlo & Sara \\
\hline MOVIMENTO 5 STELLE & $77 \%$ & $76 \%$ & $71 \%$ & $73 \%$ & $79 \%$ \\
LEGA & $47 \%$ & $62 \%$ & $57 \%$ & $48 \%$ & $52 \%$ \\
PARTITO DEMOCRATICO & $83 \%$ & $66 \%$ & $71 \%$ & $70 \%$ & $81 \%$ \\
FORZA ITALIA & $57 \%$ & $61 \%$ & $74 \%$ & $53 \%$ & $64 \%$ \\
FRATELLI D'ITALIA & $47 \%$ & $58 \%$ & $60 \%$ & $46 \%$ & $50 \%$ \\
ITALIA VIVA & $81 \%$ & $77 \%$ & $78 \%$ & $72 \%$ & $77 \%$ \\
LIBERI E UGUALI & $82 \%$ & $62 \%$ & $66 \%$ & $70 \%$ & $79 \%$ \\
+EUROPA and AZIONE & $84 \%$ & $63 \%$ & $67 \%$ & $72 \%$ & $78 \%$ \\
\hline
\end{tabular}

\section{B. Setup II}

This setup uses again the standard coding of the Likert-scale linguistic values, that is $N^{I I}=(1,2,3,4,5)$. We however allow the respondents to reflect the perceived importances of the categories of issues in the form of respondent-specific weights. This allows for the customizability of the decision support by reflecting the relative importance of each category of issues.

We still assume that the issues within one category represent "repeated measurements" of the attitude towards the overall category and as such are considered equally important within a single category. This assumption can also be relaxed, but it would require us to obtain 30 weights from each respondent, which is not feasible in reality. We also assume that the normalized weights of categories can be specified reliably by all the respondents in the form $w^{u}=\left(w_{1}^{u}, w_{2}^{u}, \ldots, w_{6}^{u}\right)$ for all $u \in R$. More specifically we have:

$$
\begin{aligned}
& \text { - } w^{\text {Melania }}=(0.33,0.27,0.20,0.07,0.07,0.07), \\
& \text { - } w^{\text {Anna }}=(0.36,0.21,0.14,0.14,0.07,0.07), \\
& \text { - } w^{\text {Marco }}=(0.25,0.25,0.13,0.13,0.13,0.13), \\
& \text { - } w^{\text {Carlo }}=(0.37,0.19,0.19,0.15,0.07,0.04), \\
& \text { - } w^{\text {Sara }}=(0.26,0.21,0.21,0.16,0.11,0.05)
\end{aligned}
$$

The (weighted) difference of the attitudes of any respondent $u \in R$ from the attitudes expressed by the party $v \in P$ can be calculated as

$$
d^{I I}\left(A^{u}, A^{v}\right)=\sum_{i=1}^{6}\left(w_{i}^{u} \cdot \sum_{j=1}^{5}\left|a_{i, j}^{u}-a_{i, j}^{v}\right|\right) .
$$

Given the normalized weights and the same number of issues in every category being assessed by the same Likert scales, we can define the maximum possible difference as $d_{\max }^{I I}=$ $\sum_{i=1}^{6} w_{i}^{u} \cdot 5 \cdot\left(\max \left(N^{I I}\right)-\min \left(N^{I I}\right)\right)=20 \sum_{i=1}^{6} w_{i}^{u}=20$ for any $u \in R$. The compatibility of the attitudes of respondent $u \in R$ to the attitudes declared by a party $v \in P$ can thus be calculated as

$$
c^{I I}\left(A^{u}, A^{v}\right)=1-\frac{d^{I I}\left(A^{u}, A^{v}\right)}{d_{\max }^{I I}} .
$$

Again $c^{I I}\left(A^{u}, A^{v}\right) \in[0,1]$ for any $u \in R$ and $v \in P$ and any vector of normalized weights $w^{u}$. Under this approach we get the compatibilities summarized in Table IV. 
TABLE IV.

SUMMARY OF THE COMPATIBILITY OF THE PREFERENCES EXPRESSED BY THE FIVE RESPONDENTS TO THE PREFERENCES EXPRESSED BY THE POLITICAL PARTIES UNDER SETUP II. FOR EACH RESPONDENT THE HIGHEST COMPATIBILITIES ARE DENOTED IN GREEN, THE LOWEST COMPATIBILITIES IN RED.

\begin{tabular}{lccccc} 
Setup II & Melania & Anna & Marco & Carlo & Sara \\
\hline MOVIMENTO 5 STELLE & $81 \%$ & $77 \%$ & $71 \%$ & $71 \%$ & $79 \%$ \\
LEGA & $46 \%$ & $63 \%$ & $57 \%$ & $46 \%$ & $48 \%$ \\
PARTITO DEMOCRATICO & $85 \%$ & $66 \%$ & $74 \%$ & $69 \%$ & $84 \%$ \\
FORZA ITALIA & $55 \%$ & $59 \%$ & $75 \%$ & $54 \%$ & $60 \%$ \\
FRATELLI D'ITALIA & $43 \%$ & $53 \%$ & $59 \%$ & $38 \%$ & $44 \%$ \\
ITALIA VIVA & $83 \%$ & $74 \%$ & $81 \%$ & $71 \%$ & $81 \%$ \\
LIBERI E UGUALI & $84 \%$ & $64 \%$ & $70 \%$ & $72 \%$ & $84 \%$ \\
\hline +EUROPA and AZIONE & $88 \%$ & $65 \%$ & $70 \%$ & $75 \%$ & $82 \%$ \\
\hline
\end{tabular}

We can see that while the parties with the lowest compatibility scores are almost the same as in Setup I, the suggested parties to vote for are different for Anna, Carlo and Sara. For all three respondents the new suggestion or the additional suggestion introduced by Setup II is such that its compatibility value in Setup I was close to the highest one in Setup I. Still, the introduction of weights changes the suggestions.

\section{Setup III}

The previous two setups used the standard coding of the linguistic values of Likert scales. From the linguistic modelling perspective, it is, however, highly unlikely that the meanings of "strongly for" and "slightly for" would have the same distance as "slightly for" and "neutral", for example. In this setup we therefore propose a different vector of numerical meanings of the linguistic terms - one that considers the "slight..." labels to be closer to the "neutral" labels than they are to the extreme labels. We define the meaning vector $N^{I I I}$ in such a way that the values remain symmetrically distributed with respect to the middle-value meaning. This way we get $N^{I I I}=(1,2 \cdot 5,3,3 \cdot 5,5)$. Even though the calibration of the meanings of the linguistic terms should be ideally done separately for each expert, we propose here a single calibration for all that at least removes the most obvious discrepancies between the linguistic values and their numerical meanings. We do not claim this is the best or optimal modification of the meaning vector - it is simply one possible modification and its effect on the final recommendations is being studied in this paper. For simplicity we do not consider the individual category weights in this setup. The difference between the attitudes of the respondent $u$ and attitudes of a party $v$ can be defined using (1), just $a_{i, j}^{u}, a_{i, j}^{v} \in\{1,2.5,3,3.5,5\}$ in this case. Because $\max \left(N^{I I I}\right)=$ $\max \left(N^{I}\right)$ and $\min \left(N^{I I I}\right)=\min \left(N^{I}\right)$, the maximum possible difference between the attitudes of a respondent and a party can again be expressed by $d_{\max }^{I}$ and (2) can be used to calculate the compatibility of the attitudes of respondent $u \in$ $R$ with the attitudes expressed by a party $v \in P$. This way we get the compatibilities summarized in Table $\mathrm{V}$.
TABLE V.

SUMMARY OF THE COMPATIBILITY OF THE PREFERENCES EXPRESSED BY THE FIVE RESPONDENTS TO THE PREFERENCES EXPRESSED BY THE POLITICAL PARTIES UNDER SETUP III. FOR EACH RESPONDENT THE HIGHEST COMPATIBILITIES ARE DENOTED IN GREEN, THE LOWEST COMPATIBILITIES IN RED.

\begin{tabular}{lccccc} 
Setup III & Melania & Anna & Marco & Carlo & Sara \\
\hline MOVIMENTO 5 STELLE & $75 \%$ & $79 \%$ & $74 \%$ & $72 \%$ & $76 \%$ \\
LEGA & $47 \%$ & $63 \%$ & $61 \%$ & $49 \%$ & $55 \%$ \\
PARTITO DEMOCRATICO & $85 \%$ & $67 \%$ & $72 \%$ & $71 \%$ & $79 \%$ \\
FORZA ITALIA & $53 \%$ & $63 \%$ & $74 \%$ & $52 \%$ & $64 \%$ \\
FRATELLI D'ITALIA & $48 \%$ & $59 \%$ & $59 \%$ & $46 \%$ & $52 \%$ \\
ITALIA VIVA & $80 \%$ & $79 \%$ & $81 \%$ & $72 \%$ & $77 \%$ \\
LIBERI E UGUALI & $82 \%$ & $62 \%$ & $66 \%$ & $71 \%$ & $76 \%$ \\
+EUROPA and AZIONE & $85 \%$ & $64 \%$ & $68 \%$ & $74 \%$ & $76 \%$ \\
\hline
\end{tabular}

The different assignment of numerical meanings to the linguistic values of the Likert scale here results in just one suggestion of a party to vote for that is being changed (Carlo), but for two respondents (Melania and Anna) the new setup introduces a second party suggestion that is equally compatible as the one suggested in Setup I. Also, for Marco the suggestion of the least compatible party is different than in Setup I.

\section{D.Setup IV}

In this case we investigate what happens with the suggestion provided by our decision-support model, if we remove the potential leniency/central-tendency effects in the data by grouping together the answers provided in the positive direction ("strongly for" and "slightly for"), the answers provided in the neutral direction ("neutral"), and the answers provided in the negative direction ("slightly against" and "strongly against"). This can be achieved by defining the vector of numerical meanings of the linguistic values of the Likert scale, for example, as $N^{I V}=(1,1,3,5,5)$.

The difference between the attitudes of the respondent $u$ and attitudes of a party $v$ can again be defined using (1), just $a_{i, j}^{u}, a_{i, j}^{v} \in\{1,3,5\}$ in this case. Because of the choice of $N^{I V}$ we again have $\max \left(N^{I V}\right)=\max \left(N^{I}\right)$ and $\min \left(N^{I V}\right)=$ $\min \left(N^{I}\right)$, the maximum possible difference between the attitudes of a respondent and a party can again be expressed by $d_{\max }^{I}$ and (2) can be used directly to calculate the compatibility of the attitudes. Note that the actual numerical values used in the vector $N^{I V}$ do not matter as long as the three numerical values assigned are ordered and the minimum has the same distance from the middle value as the maximum does. In other words (2) gives in this case the same result for any alternative definition of the vector $N^{I V}=(b-a, b-$ $a, b, b+a, b+a)$ for any two real numbers $a$ and $b, a>0$. The compatibilities of the attitudes of the respondents and the parties under this setup are summarized in Table VI.

This Setup also changes the initial suggestions of the parties to vote for with respect to Setup I - Melania is suggested a second option, Sara two additional (equally compatible) options and Marco is suggested a different party. In terms of least compatible parties Anna is now left with two such parties while Melania with only one. 
TABLE VI.

SUMMARY OF THE COMPATIBILITY OF THE PREFERENCES EXPRESSED BY THE FIVE RESPONDENTS TO THE PREFERENCES EXPRESSED BY THE POLITICAL PARTIES UNDER SETUP IV. FOR EACH RESPONDENT THE HIGHEST COMPATIBILITIES ARE DENOTED IN GREEN, THE LOWEST COMPATIBILITIES IN RED.

\begin{tabular}{|c|c|c|c|c|c|}
\hline Setup IV & Melania & Anna & Marco & Carlo & Sara \\
\hline MOVIMENTO 5 STELLE & $80 \%$ & $70 \%$ & $65 \%$ & $75 \%$ & $85 \%$ \\
\hline LEGA & $45 \%$ & $62 \%$ & $50 \%$ & $47 \%$ & $47 \%$ \\
\hline PARTITO DEMOCRATICO & $80 \%$ & $63 \%$ & $68 \%$ & $68 \%$ & $85 \%$ \\
\hline FORZA ITALIA & $63 \%$ & $57 \%$ & $75 \%$ & $55 \%$ & $65 \%$ \\
\hline FRATELLI D'ITALIA & $47 \%$ & $57 \%$ & $62 \%$ & $45 \%$ & $45 \%$ \\
\hline ITALIA VIVA & $82 \%$ & $72 \%$ & $73 \%$ & $73 \%$ & $77 \%$ \\
\hline LIBERI E UGUALI & $80 \%$ & $63 \%$ & $65 \%$ & $68 \%$ & $85 \%$ \\
\hline +EUROPA and AZIONE & $82 \%$ & $62 \%$ & $63 \%$ & $70 \%$ & $73 \%$ \\
\hline
\end{tabular}

\section{E. A linguistic fuzzy modelling interface for decision support}

It is clear from the comparison of results presented in Tables III to VI that even though the differences in the ordering of parties with respect to their compatibility with the respondents (in terms of attitudes to the important topics and their categories) can be found for all the respondents across the four setups, the relative differences in the compatibility values are rather small. Moreover, in many cases a difference of one or a just few percentage points determines which party will be suggested as the most compatible one. As such the models (setups) can be considered sensitive even to a single answer - note that if the total maximum difference is 120 for the non-weighted models, then a difference of two levels of the linguistic assessment (numerically a difference of 2) can already result in a $1 \%$ difference in compatibility.

This does not mean that the models would not be useful. It might, however, be a good idea to accompany the suggestion of a "most compatible" and "least compatible" party by a piece of information on a completely different level of granularity. We therefore propose here an additional linguistic fuzzy modelling based tool, that helps the respondents answer a more general question - "Should I consider voting for a given party?" This can be considered a question on sufficient compatibility of the attitudes of the respondent and the given party. In other words, this question does not ask for the ordering of the parties in terms of their compatibility. It is more of an absolute-type evaluation question aiming to identify which parties are "compatible enough". As a consequence, the answer to this question does not need to distinguish between the parties that are considered compatible enough and might not offer their ordering. We also add the opposite perspective and ask "Should I avoid voting for this party?" - with similar reasoning this is a question looking for too large a difference in the attitudes of the respondent and the party in order for the party to still constitute a reasonable choice for the respondent.

To get the necessary answers to these questions we will focus on the categories of issues and define an "acceptable difference in attitudes" $A D A_{u}^{C_{i}}$ of a respondent $u \in R$ in the category of important issues $C_{i}$ as a trapezoidal fuzzy set on the universe $[0,20]$, where $20=d_{\text {max }}^{C_{i}}$ is the largest possible total difference in the numerical values of the assessments of attitudes towards the respective five important issues in category $C_{i}, i=1, \ldots, 6$. In other words the membership function of the fuzzy set $A D A_{u}^{C_{i}}$ (denoted for simplicity $\left.A D A_{u}^{C_{i}}(x)\right)$ maps $[0,20]$ into $[0,1]$ such that for any $x \in$ $[0,20]$ the value $A D A_{u}^{C_{i}}(x)$ represents the extent of acceptability of that particular size of difference (1 meaning fully acceptable and 0 meaning $0 \%$ acceptable). For simplicity we will use trapezoidal-shaped membership functions that can be fully characterized by 4 characteristic values $k, l, m, n \in[0,20]$ such that $k \leq l \leq m \leq n$, $A D A_{u}^{C_{i}}(x)=0$ for all $x \in[0, k] \cup[n, 20], A D A_{u}^{C_{i}}(x)=1$ for all $x \in[l, m]$ and $A D A_{u}^{C_{i}}(x)$ is linear between $k$ and $l$ and also between $m$ and $n$. In this case we write $A D A_{u}^{C_{i}} \sim(k, l, m, n)$. We will also use the minimum triangular norm to represent the intersection of fuzzy sets (and thus the logical conjunction of their linguistic meanings) and the maximum triangular conom to represent the union of fuzzy sets (and thus the logical disjunction of their linguistic meanings). See [16,17] for more details on fuzzy set theory.

Let $A D A_{u}^{C_{i}} \sim\left(k_{u}^{i}, l_{u}^{i}, m_{u}^{i}, n_{u}^{i}\right)$ be a trapezoidal fuzzy number representing the acceptable values of the difference between the attitudes of the respondent and a given party with respect to category $C_{i}$ defined by (valid for) the respondent $u \in R$, $i=1, \ldots, 6$. In this case it is reasonable to expect that $k_{u}^{i}=$ $l_{u}^{i}=0$. Let $U D A_{u}^{C_{i}} \sim\left(K_{u}^{i}, L_{u}^{i}, M_{u}^{i}, N_{u}^{i}\right)$ be a trapezoidal fuzzy number representing the unacceptable values of the difference in attitudes of the respondent and a given party with respect to category $C_{i}$ defined by (valid for) the respondent $u$. Let (5) define the numerical value of a difference in attitudes in category $C_{i}$ between the respondent $u \in R$ and a party $v \in P$. In this case we would expect that $M_{u}^{i}=N_{u}^{i}=d_{\max }^{I}$ for all $i=1, \ldots, 6$. Then the overall strength supporting the claim "Respondent $u$ should consider voting for party $v$." can be calculated as support $t_{u}^{v} \in[0,1]$ using (6).

$$
d^{C_{i}}\left(A^{u}, A^{v}\right)=\sum_{j=1}^{5}\left|a_{i, j}^{u}-a_{i, j}^{v}\right|
$$

$\operatorname{support}_{u}^{v}=$

$$
=\min \left\{A D A_{u}^{C_{1}}\left(d^{C_{1}}\left(A^{u}, A^{v}\right)\right), \ldots, A D A_{u}^{C_{6}}\left(d^{C_{6}}\left(A^{u}, A^{v}\right)\right)\right\}
$$

Formula (6) represents the requirement of the distances in attitudes being acceptable in all the categories at the same time. The overall strength supporting the claim "Respondent $u$ should avoid voting for party v." can be calculated as $\operatorname{avoid}_{u}^{v} \in[0,1]$ using (7):

$\operatorname{avoid}_{u}^{v}=$ $=\max \left\{U D A_{u}^{C_{1}}\left(d^{C_{1}}\left(A^{u}, A^{v}\right)\right), \ldots, U D A_{u}^{C_{6}}\left(d^{C_{6}}\left(A^{u}, A^{v}\right)\right)\right\}$.

This way (7) represents the idea that if at least one of the categories is such that the difference in preferences there is unacceptable, then one should avoid voting for that party.

Note that the definitions of the fuzzy numbers $A D A_{u}^{C_{i}}$ and $U D A_{u}^{C_{i}}$ substitute the need for the definitions of weights of the categories and directly reflect the requirements on the strength of compatibility of the respondent with the party in terms of the attitudes towards a given category of criteria. For 
example, the closer the interval $\left[m_{u}^{i}, n_{u}^{i}\right]$ is to the left side of the $[0,20]$ interval, the more important the compatibility (agreement) in this category is for the respondent.

Let us now see what kind of decision support such an approach can provide. If Melania's definitions of the fuzzy numbers representing the acceptable values of the differences in the categories are:

- $A D A_{\text {Melania }}^{C_{1}} \sim(0,0,4,10)$,

- $\quad A D A_{\text {Melania }}^{C_{2}} \sim(0,0,6,12)$,

- $A D A_{\text {Melania }}^{C_{3}} \sim(0,0,6,12)$,

- $A D A_{\text {Melania }}^{C_{4}} \sim(0,0,8,14)$,

- $A D A_{\text {Melania }}^{C_{5}} \sim(0,0,8,14)$,

- $A D A_{\text {Melania }}^{C_{6}} \sim(0,0,8,14)$,

and the fuzzy numbers representing unacceptable values of differences in the categories are:
- $U D A_{\text {Melania }}^{C_{1}} \sim(10,16,20,20)$,
- $U D A_{\text {Melania }}^{C_{2}} \sim(12,16,20,20)$,
- $U D A_{\text {Melania }}^{C_{3}} \sim(12,16,20,20)$,
- $U D A_{\text {Melania }}^{C_{4}} \sim(14,18,20,20)$,
- $U D A_{\text {Melania }}^{C_{5}} \sim(14,18,20,20)$,
- $U D A_{\text {Melania }}^{C_{6}} \sim(14,18,20,20)$,

then the following decision-support would be provided. Decision support is formulated linguistically as an answer to the original question, the number in bracket represents the support $_{u}^{v}$ value:

- Consider voting for Movimento 5 Stelle (100\%),

- Consider voting for +Europa e Azione (100\%).

- Consider voting for Italia Viva (100\%),

- Consider voting for Partito Democratico (83\%),

- Consider voting for Liberi e Uguali (50\%), and also (now values in brackets represent the avoid $u_{u}^{v}$ values):

- Avoid voting for Fratelli d'Italia (100\%),

- Avoid voting for Lega (66\%), and

- Avoid voting for Forza Italia (33\%).

We can see that those parties with overall high compatibilities in Setups I-IV are suggested for consideration while those that were scored frequently as incompatible are suggested to be avoided. This approach does not provide a clear answer to the question whom to support, but it seems to be able to summarize the situation reasonably well, to provide linguistic outputs and to cover the information obtained through the use of setups I-IV. It is definitely an approach to consider at least as an additional source of information for informed choice in the election situation. Note that linguistic summaries are being applied more and more often recently $[18,19]$ because of their easy understandability by laymen.

\section{DISCUSSION}

Setups I-IV investigate different possible uses of a 5-point Likert scale for the assessment of attitudes of parties and respondents towards a given set of thirty important issues grouped into six categories. The important take-away message of these approaches is the fact that there were differences in the suggestion of the most compatible and least compatible party at least for one of the respondents between each pair of setups. This implies that the choice of the setup has to be done correctly - mainly the coding of the linguistic values and the need for the reflection of perceived importances of the issues (or categories thereof) by the respondents, potentially the need for countermeasures to the central tendency/leniency issue need to be well thought through. As such the choice of the setup to provide correct voters' decision support and also correct research data for political sciences is not trivial. All the four setups considered in this paper define the suggestion of a party to vote for based on the maximum compatibility score, that might have a very close runner up with only a slightly lower compatibility score, which is then, however, discarded. The setups thus seem to be very sensitive to the precision of the actual values provided by the respondents and the experts assessing the programs of the parties.

One can also argue that the very goal of the decision support is not specified well in the four setups. How reasonable is it to look for the most compatible party (in terms of attitudes to the important issues)? First of all, we need to understand that this goal calls for a relative-type evaluation. This type of evaluation is, by definition, dependent on the set of available parties and also on the actual values of compatibilities. If the set of parties does not contain all the parties to be considered, the relative-type decision support can be biased. There is also one more potential, and well known, issue connected with relative type evaluation and decision support based on such evaluation - the inability of the model to assess whether the best choice that is to be presented to the decision-maker as a decision-support is "good enough" to be accepted. The most compatible party might still not be a party to vote for, if its compatibility with one's attitudes is low. Unfortunately, if the goal is formulated in terms of finding the "most compatible" party, then the answer we are getting is formally correct, even though it might not be practically correct or relevant. One should therefore at least make sure that the set of parties is complete. Then it might be justifiable to accept that the most compatible party is the one to vote for, as there is no better one available. Still, it seems that not knowing whether the suggested party is "compatible enough" with the voter to choose that party in reality can seriously bias the research based on such data.

We have therefore suggested a linguistic fuzzy modelling based approach to the assessment of the available data. The proposed linguistic fuzzy modelling based tool allows for the definition of an acceptable magnitude of difference in attitudes and also for the definition of an unacceptable (too high) magnitude of difference in preferences. Having done so the respondent (or the researcher conducting a research on voter preferences or assumed choices) can be provided with a list of parties that are "sufficiently close" in terms of the attitudes to the categories of important issues to be considered for selection and also with a list of parties that "differ in their attitudes too much" to be voted for. Both that with the 
measure of support or strength of that suggestion represented by the $\operatorname{support}_{u}^{v}$ and avoid $v$ values respectively.

Clearly the linguistic approach provides a different perspective on the voters' potential choices than the usual models aiming on suggesting the most fitting solution. Instead of a single party with the best compatibility (which can be the best by a very slim margin) it lists all the parties that are "acceptable enough". The other side of the universe is also covered by listing those parties that are "too different in their attitudes towards the important issues" to be voted for.

It is also interesting to note that the linguistic fuzzy modelling perspective offers more customizability to the decision support. First, the membership functions of the fuzzy numbers that represent acceptable and unacceptable values of the differences are defined in an absolute way. This means that the respondent can define the thresholds for (un)acceptable values of differences prior to the very task of data input, independently of the already available data. The information is provided in the units of the distance of the attitudes (expressed numerically) and as such these units can be expected to be well understood by the respondent/voter. It might also mean that the (un)acceptability thresholds required here (i.e. the definitions of the membership functions of the fuzzy numbers that represent (un)acceptable magnitudes of differences in attitudes) are much easier to define than abstract unitless weights of the categories. And as we have discussed previously, the fuzzy numbers defined for this purpose essentially perform a similar function as the weights would perform for example in the Setup II.

Second, the aggregation using the min t-norm and the max tconorm respectively does not allow for compensation, unlike the approaches relying on (weighted) sums or (weighted) arithmetic means. In other words, the model is built in such a way that a clear incompatibility in a single category of important issues (the difference in attitudes is acceptable with the strength of 0 ) results in the strength of the suggestion to vote for that party to be $0 \%$ regardless of the compatibility in the other categories of important issues. Analogously the suggestion not to vote for a party is provided with the strength of $100 \%$ if the difference in attitudes is considered $100 \%$ unacceptable in a single category of important issues regardless of the compatibility in the other categories. This is a rather strict approach, that can still be customized by the choice of different t-norms and t-conorms. In any case it reflects a risk-averse approach to the evaluation of the compatibility of the attitudes of respondents and parties and as such it provides a good benchmark to the other models. Alternatively, one could also consider representing the attitudes of the parties and the respondents including the perceived relevance of the issues and apply the tools of interval-valued semantic differential [20] and see which parties are the closest in the semantic space in terms of the ndimensional representation of their attitudes. This however remains out of the scope of this particular paper.

\section{V.CONCLUSION}

The paper investigated the possibility of constructing a voter decision-support using surveys with Likert-type answers. Based on the studied Setups we can conclude that the use of calibrated and non-calibrated Likert scales can result in a different suggestion (decision-support being provided). The inclusion of weights of categories of important issues also influences the results of the decision support. Lastly switching to the $+/ 0 /$ - understanding of the values of the Likert scales also changes the decision support provided by the models. This all implies that if a calibration of the Likert scale is needed (i.e. if its linguistic terms cannot be considered equidistant) or if the categories have different perceived importance for the choice to be made by the voter, then these need to be reflected in the model. Otherwise, the decision-support can be biased. We have also proposed a linguistic fuzzy modelling interface for the evaluation of the data obtained through Likert scales that allows for the reflection of voters' preferences and priorities by the definitions of (un)acceptable values of differences. These definitions have the benefit of being absolute-type (i.e. they directly specify what values are acceptable and what values are not, independently of the actual available data), no standardization is needed), they can be expressed directly in the units of the magnitude of difference and also introduce "(un)acceptability thresholds" above or below which the parties are no longer discriminated and considered "too different" or "compatible enough" to be either discarded or considered viable choices. This reduces the potentially undesirable sensitivity of the model to minor changes in the answers provided by the Likers scales. Even though the definition of the fuzzy-number representations of the (un)acceptable magnitudes of differences in attitudes might be slightly more demanding for the voter, we still strongly recommend this approach to be able to obtain a less sensitive and well understandable decision support in the voting process. And also, to obtain a fresh and novel type of data for the pre-election surveys and analytics.

\section{ACKNOWLEDGEMENT}

The authors would like to express their thanks to Douglas Adams [21] for the inspiration for the title of the paper.

\section{REFERENCES}

[1] S. R. Muller, and C. E. Thomas, "Election Infrastructure Security: Grants and Reimbursement to the States for Usage of their National Guards in State Active Duty Status to Provide Cybersecurity for Federal Elections," in Proceedings of the 2020 International Conference on Research in Management \& Technovation, Shivani Agarwal, Darrell Norman Burrell, Vijender Kumar Solanki (eds). ACSIS, Vol. 24, pp. 73-78, 2020, doi: 10.15439/2020KM7

[2] S. P. Robertson, "Voter-centered design: Toward a Voter Decision Support System," ACM Trans. Comput. Interact., vol. 12, no. 2, pp. 263-292, 2005, doi: 10.1145/1067860.1067866.

[3] R. Likert, "A technique for the measurement of attitudes," Arch. Psychol., vol. 22, no. 140, pp. 5-55, 1932.

[4] J. C. Rogowski, "Voter Decision-Making with Polarized Choices," Br. J. Polit. Sci., vol. 48, no. 1, pp. 1-22, 2018, doi: $10.1017 / \mathrm{S} 0007123415000630$. 
[5] J. Stoklasa, T. Talášek, and J. Stoklasová, "Semantic differential for the twenty-first century: scale relevance and uncertainty entering the semantic space," Qual. Quant., vol. 53, no. January 2019, pp. 435448, May 2019, doi: 10.1007/s11135-018-0762-1.

[6] J. Stoklasa, T. Talášek, and J. Stoklasová, "Reflecting emotional aspects and uncertainty in multi-expert evaluation: one step closer to a soft design-alternative evaluation methodology," in Advances in Systematic Creativity: Creating and Managing Innovations, L. Chechurin and M. Collan, Eds. Palgrave Macmillan, 2019, pp. 299322.

[7] J. Stoklasová, T. Talášek, and J. Stoklasa, “Attitude-based multiexpert evaluation of design," in Intelligent Systems and Applications in Business and Finance, P. Luukka and J. Stoklasa, Eds. Springer, (in press).

[8] G. Hoang, J. Stoklasa, and T. Talášek, "First steps towards a lossless representation of questionnaire data and its aggregation in social science and marketing research," in Proceedings of the international scientific conference Knowledge for Market Use 2018, 2018, pp. 112118.

[9] J. Stoklasa, T. Talášek, J. Kubátová, and K. Seitlová, "Likert scales in group multiple-criteria evaluation," J. Mult. Log. Soft Comput., vol. 29, no. 5, pp. 425-440, 2017.

[10] J. Stoklasa, T. Talášek, and P. Luukka, "Fuzzified Likert scales in group multiple-criteria evaluation," in Soft Computing Applications for Group Decision-making and Consensus Modeling, vol. 357, M. Collan and J. Kacprzyk, Eds. Springer International Publishing AG, 2018, pp. 165-185.

[11] G. Norman, "Likert scales, levels of measurement and the 'laws' of statistics," Adv. Heal. Sci. Educ., vol. 15, no. 5, pp. 625-632, 2010.

[12] A. Furnham, "Response Bias, Social Desirability Dissimulation," Pers. Individ. Dif., vol. 7, no. 3, pp. 385-400, 1986.
[13] A. Furnham and M. Henderson, "The good, the bad and the mad: Response bias in self-report measures," Pers. Individ. Dif., vol. 3, no. 3, pp. 311-320, 1982.

[14] J. Stoklasa and T. Talášek, "On the use of linguistic labels in AHP: calibration, consistency and related issues," in Proceedings of the 34th International Conference on Mathematical Methods in Economics, 2016, pp. 785-790.

[15] J. Stoklasa, Linguistic models for decision support. Lappeenranta: Lappeenranta University of Technology, 2014.

[16] G. J. Klir and B. Yuan, Fuzzy Sets and Fuzzy Logic: Theory and Aplications. New Jersey: Prentice Hall, 1995.

[17] D. Dubois and H. Prade, Eds., Fundamentals of Fuzzy Sets. Massachusetts: Kluwer Academic Publishers, 2000.

[18] J. Stoklasa, T. Talášek, and J. Stoklasová, "Executive summaries of uncertain values close to the gain/loss threshold - linguistic modelling perspective," Expert Syst. Appl., vol. 145, p. 113108, 2020, 10.1016/j.eswa.2019.113108

[19] Ł. Sosnowski, and T. Penza, "Generating Fuzzy Linguistic Summaries for Menstrual Cycles," Proceedings of the 2020 Federated Conference on Computer Science and Information Systems, M. Ganzha, L. Maciaszek, M. Paprzycki (eds). ACSIS, Vol. 21, pp. 119128, 2020, doi: 10.15439/2020F202

[20] J. Stoklasová, "Interval-valued semantic differential in multiple criteria and multi-expert evaluation context: possible benefits and application areas" Recent Advances in Business Analytics. Selected papers of the 2021 KNOWCON-NSAIS workshop on Business Analytics, Jan Stoklasa, Pasi Luukka and Maria Ganzha (eds). ACSIS, Vol. 29, pp. 53-61, 2021, doi: 10.15439/2021B3

[21] D. Adams, The Hitchhiker's Guide to the Galaxy. New York: Harmony Books, 1980. 